\title{
Népal. Rituel et Divertissement
}

Enregistrements (1995-1996). Patrimoine Musical - Musiques

Traditionnelles d'Aujourd'hui. Auvidis/Naïve, 1999

\section{Franck Bernède}

\section{(2) OpenEdition}

12 Journals

Édition électronique

URL : http://journals.openedition.org/ethnomusicologie/774

ISSN : 2235-7688

Éditeur

ADEM - Ateliers d'ethnomusicologie

Édition imprimée

Date de publication : 1 janvier 2001

Pagination : 260-263

ISBN : 2-8257-0723-6

ISSN : 1662-372X

Référence électronique

Franck Bernède, « Népal. Rituel et Divertissement », Cahiers d'ethnomusicologie [En ligne], 13 | 2001, mis en ligne le 09 janvier 2012, consulté le 19 avril 2019. URL : http://journals.openedition.org/ ethnomusicologie/774

Ce document a été généré automatiquement le 19 avril 2019.

Tous droits réservés 


\title{
Népal. Rituel et Divertissement
}

\author{
Enregistrements (1995-1996). Patrimoine Musical - Musiques \\ Traditionnelles d'Aujourd'hui. Auvidis/Naïve, 1999
}

\section{Franck Bernède}

\section{RÉFÉRENCE}

Népal. Rituel et Divertissement. Enregistrements (1995-1996), textes et photos: Sophie Laurent. Patrimoine Musical - Musiques Traditionnelles d'Aujourd'hui. Avec le soutien de la Faculté de musique de l'Université de Montréal. Collection UNESCO. 1 CD Auvidis/ Naïve D8279, 1999.

1 L'Himalaya se caractérise par un foisonnement de sociétés et de cultures dont la pluralité des expressions artistiques reflètebien la richesse. Situé entre les deux géants que sont l'Inde et la Chine, le royaume du Népal est principalement influencé, du point de vue socio-religieux, par les valeurs de l'hindouisme et du système des castes; les différentes formes du bouddhisme, représentées par les communautés néwar, tibétaine et certainesethnies tibéto-birmanes en présence, n'étant pratiquées que par une fraction relativement restreinte de la population. Parmi les castes indo-népalaises, le savoir musical est une activité partagée par des groupes semi-professionnels, classés selon le code législatif népalais au rang social le plus bas. Parmi eux, les castes de tailleursmusiciens Damâi et de ménestrels Gâiné sont présentes sur l'ensemble du territoire et leurs traditions régionales étaient encore peu connues.

Cette nouvelle production discographique comporte six parties organisées par aires géoculturelles; la première est consacrée aux tailleurs-musiciens Damâi, la seconde aux ménestrels Gâiné, alors que la troisième s'attache à certains aspects de la musique et de la danse des Néwar de la vallée de Kathmandu; le panorama est complété par un aperçu du répertoire des Tharu, des Sherpa et de plusieurs musiques villageoises du piémont.

3 L'aspect le plus remarquable de ce travail est sans nul doute celui qui concerne les tailleurs-musiciens Damâi, auxquels l'auteur consacre une part importante (plages 1 à 7). 
Sensibilisée aux contours stylistiques contrastés de la forme musicale dominante de leur répertoire, le pa-ca bâjâ, Sophie Laurent nous convie à une appréhension esthétique des variations régionales de cet ensemble emblématique de la culture népalaise. Si cette présentation comparative ne prétend pas à l'exhaustivité, elle nous laisse entrevoir la richesse de cette musique à travers sept exemples représentatifs collectés dans l'est, le centre et l'ouest du pays ${ }^{1}$. L'atmosphère restituée dans ces enregistrements par la conjonction des timbres et des dynamiques, évoque avec force la bipolarité des fonctions musicales (rituelles et festives) dans un environnement où prêtriseet royauté s'interpénètrent sur un territoire entendu comme essentiellement rituel $^{2}$. Les deux premières plages, consacrées à l'ensemble nagarâ bânâ, assigné au palais royal de Gorkha et au sanctuaire de la déesse Manakâmanâ, en sont des témoignages éminents. D'un ton plus léger, l'exécution du râg bela contraste avec les pièces qui précèdent. Le câcari qui suit, bien que joué en contexte rituel, montre à l'envi le caractère de divertissement que peut également revêtir l'ensemble pa-ca bâjâ. La plage 5, enregistrée dans l'est du pays, fait bien ressortir l'importance accordée au jeu du tambour dholak dans l'organisation rythmique de la pièce. Quant aux deux autres exemples de cette première sélection, ils montrent la variabilité des styles en usage. On notera en particulier avec l'auteur l'emphase mise sur le jeu des timbales damâhâ dans la plage 6 et les modifications de timbre de la trompe karnâl dans l'exécution d'un rituel domestique à la plage 7.

Dans le paysage sonore himalayen, largement dominé par les membranophones et les aérophones, les ménestrels Gâiné (plage 8) sont, avec les tanneurs-musiciens Bâdi, les deux seuls groupes à pratiquer des instruments à cordes frottées (deux types de vièles sarangi) $)^{3}$. Parmi les thèmes narratifs traditionnellement exprimés par les Gâiné (chants lyriques, religieux, hérö̈ques et militaires), Sophie Laurent nous propose l'écoute d'un chant lyrique lok git jyâure, enregistré dans l'ancienne capitale de Gorkha (centre-ouest du pays). Hormis l'évocation de la richesse narrative, des métaphores, des rimes et du langage onomatopéique des récits pour lesquels on eût aimé avoir accès à une traduction dans le livret, on notera la technique originale du jeu de l'instrument, qui est l'un des pôles d'attraction de cette pièce: pizzicati de main gauche, associés à un coup d'archet qui ressemble singulièrement au saltendo pratiqué par les violonistes occidentaux et différents types de trilles, utilisés comme ornements de la phrase musicale, qui rappellent ceux de la voix chantée dans nombre de traditions himalayennes et dont le jeu de la vièle se fait ici l'écho.

5 L'extraordinaire diversité de la tradition musicale et chorégraphique des Néwar de la vallée de Kathmandu n'est plus à démontrer. Elle a déjà fait l'objet de plusieurs présentations discographiques partielles (cf. Hoerburger 1971, Aubert 1989, Bernède 1997b). Elle est illustrée ici par trois pièces caractéristiques. La plage 9, dévolue à l'exécution d'un Mâ par l'ensemble de tambours dhimay du Département de Musicologie de l'Université de Kathmandu (dirigé par le Prof. Gert Mathias Wegner), présente une démonstration éclatante des possibilités de la formation. Elle reflète néanmoins un état de sophistication rarement entendu dans l'exécution de ce répertoire lors des manifestations rituelles et festives de la communauté des paysans Maharjan, traditionnellement dépositaire de cette tradition. Au moment où la pratique des hautbois mvâli tend à disparaître complètement du paysage musical néwar, l'enregistrement d'un phâgu (plage 10) interprété par les tailleurs-musiciens néwar Jogi du célèbre sanctuaire de Changu Narayan, est particulièrement bienvenue. Les «musiques de scène» accompagnant la danse Nârada nâc (Kartik pyâkham en néwari) exécutée chaque année 
devant les fenêtres de l'ancien palais royal de Patan est un exemple édifiant des ramifications normatives entre rites et fêtes chez les Newar. L'extrait choisi (plage 11) provient du tableau final de l'exécution. Il laisse clairement entendre la technique particulière du jeu des trompettes naturelles pvomgâh, l'un des aérophones dominants dans le répertoire rituel newar.

6 En nous conviant à découvrir le monde sonore des Tharu, un groupe ethnique important (plus d'un million de personnes) disséminé sur l'ensemble des basses terres du Népal et du Kumaon indien, Sophie Laurent lève le voile sur l'un des domaines encore peu exploré des musiques himalayennes. En effet, si les différents groupes Tharu sont maintenant bien circonscrits au plan anthropologique, leurs pratiques artistiques (musiques et danses) n'ont jamais été abordées sous l'angle ethnomusicologique. Il convient donc de saluer ici l'initiative de l'auteur. L'exécution d'une danse de bâton ${ }^{4}$ (plage 13), effectuée aux abords du parc National de Chitwan, haut lieu du tourisme au Népal, est un témoignage sociologique montrant l'usage commercial de cette danse traditionnelle. On sera plus sensible aux deux (trop) courts extraits de chant de femmes et de chant alterné qui suivent (plages 14 et 15). Ces derniers reflètent bien l'atmosphère des mariages et des fêtes villageoises. Le bref aperçu de l'art des Sherpa qui suit (plage 16), enregistré à Kathmandu auprès de jeunes de cette communauté, semble peu représentatif de leur culture $^{5}$. On appréciera en revanche la sélection des musiques indo-népalaises variées (plages 17 à 19) qui forme la dernière partie de ce disque.

7 La compréhension des musiques himalayennes implique une approche pluridisciplinaire et requiert bien souvent (pour ne pas dire toujours) de la part de l'ethnomusicologue des connaissances approfondies relevant de disciplines comme l'indologie, l'anthropologie, la linguistique ou encore l'épigraphie. Sur ce plan, le contenu du livret d'accompagnement de ce CD laisse transparaître quelques faiblesses qui peuvent prêter à la critique. On notera par exemple la confusion entre le siddha Gorakhnâth et la déesse Kâlikâ (commentaire de la plage 1). Gorakhnâth n'est pas une déesse, mais le fondateur d'une lignée ascético-yogique, les Gorakhnâthî, appelés également Kânphatâyogi («ceux aux oreilles fendues»). Il est conjointement vénéré avec la déesse Kâlikâ, divinité lignagère de la dynastie des Shah de Gorkha. Dans ce registre, on émettra également quelques réserves quant à l'utilisation interchangeable des termes bardes/ ménestrels utilisés ici pour désigner les Gainé. Il paraît plus approprié de les nommer simplement ménestrels et de réserver le terme barde à des musiciens récitant des épopées(comme les Hudkiyâ du Kumaon indien ou les Hudke-Damâi de l'extrême ouest du Népal). Au plan musicologique, on aurait aimé avoir quelques informations supplémentaires sur l'origine du râg bela. et du mode rythmique hori tâla (plages 3 et 10).

8 L'approche méthodologique du travail de Sophie Laurent offre, au-delà d'une accessibilité «tout public», un matériel de choix aux chercheurs intéressés par l'étude comparée des styles, domaine encore peu exploré. Cet éclairage est facilité par la durée conséquente des plages qui incite à une plongée dans le monde sonore proposé. On mentionnera enfin la grande qualité des enregistrements réalisés et en particulier le soin mis à une bonne spatialisation de l'événement sonore. A un moment charnière de l'histoire musicale du Népal, où nombre de traditions disparaissent ou subissent d'irréversibles processus de folklorisation, ce disque est un témoignage important pour l'évaluation d'un milieu en pleine mutation (le cas du mvâli bâjâ newar est particulièrement représentatif à cet égard). Cette première publication discographique de Sophie Laurent est donc prometteuse et nous ne pouvons que l'encourager à poursuivre dans une voie où le cercle 
des ethnomusicologues himalayistes se fait malheureusement de plus en plus restreint. Espérons que cette excellente publication générera des vocations.

\section{BIBLIOGRAPHIE}

BERNÈDE Franck, ed., 1997a, Himalayan Music, State of the Art. A special double issue of the European Bulletin of Himalayan Research, Paris: CNRS; London: SOAS; Heidelberg: SAI.

HELFFER Mireille, 1966, «Sur un sarangi de Gâiné». Objets et mondes VI/2: 133-141.

HELFFER Mireille, 1968, «Remarques sur le vers népali chanté». L’Homme VIII/4: 37-91.

HELFFER Mireille, 1977, «Une caste de musiciens: les Gâiné du Népal». L’Ethnologie 73: 47-75.

LAURENT Sophie, 1997, «The Music at Manakâmanâ Temple: Essay on the Style of Ritual Music of Damâi Musicians». In Bernède 1997a.

TINGEY Carole, 1994, Auspicious Music in a Changing Society. The Damâi musicians of Nepal. London: SOAS (Musicology Series 2).

\section{Références discographiques}

AUBERT Laurent, 1989, Népal. Musique de fête chez les Newar. Enregistrements réalisés en 1952 par Marguerite Lobsiger-Dellenbach (2) et en 1973 par Laurent Aubert (1, 3-17). Livret d'accompagnement de Laurent Aubert. 1 CD Archives internationales de musique populaire, AIMP XIII / VDE-GALLO CD-553.

BERNÈDE Franck, 1997b, Bardes de l'Himalaya, Epopées et Musique de Transe. Collection CNRS / Musée de L'Homme. 1 CD Le Chant du Monde CNR 2741080.

HELFFER Mireille, 1969, Castes de musiciens au Népal. Enregistrements de M. Gaborieau, M. Helffer, C. Jest et A.W. Macdonald. Livret d'accompagnement de Mireille Helffer. Paris: Musée de l'Homme, Editions du Département d'Ethnomusicologie LD20 (épuisé).

HOERBURGER Felix, 1971, Nepal. Musik der Nevârî Kasten. 1 LP Berlin: Klangdokumente zur Musikwissenschaft KM 0003.

VALENTIN Serge, s.d., Musique traditionnelle du Népal. Gaïnés de Hyangja, région de Pokhara. $1 \mathrm{CD}$, Musique du Monde. Buda Records 82493-2.

WEGNER Gert Mathias, 1999, Music of The Sherpa People of Nepal I: Shebru Dance-Songs from the Everest Region. Kathmandu/ Salzburg. 2 CD Eco Himal.

WEISETHAUNET H., 1977, The Real Folk Music of Nepal. Performance and Narratives of the Wandering Gâine Musicians. 1 CD Travelling Records DDD TR-001-2. 


\section{NOTES}

1. Pour une présentation de cet ensemble dans l'extrême Ouest du Népal et le Kumaon indien, cf. Bernède 1997b.

2. Sur les relations entre musique, pouvoir et territoire en Himalaya, cf. Carol Tingey: «Music for the Royal Dasai (Gorkhâ and Nuwâkot)» in Bernède 1997a, et Gérard Toffin: «Le tambour et la ville. De l'ethnomusicologie à l'anthropologie urbaine (Népal)», L'Homme 146, 1998: 113-142.

3. Mentionnons en passant deux traditions pour ainsi dire obsolètes dans l'Himalaya du Népal, celle des vièles à archet emprisonné (au Mustang et chez les Newar), héritage probable de la chine.

4. Les «danses de bâtons», sont l'un des traits culturels partagés par différentes communautés du Népal. Celle de la communauté Néwar de Bhaktapur, exécutée avec faste durant la fête la vache ( Gâi jatra), est des plus démonstratives.

5. On consultera, à des fins comparatives, la récente publication d'un double CD sur la musique de ce groupe (Wegner 1999). 